\title{
Experiences of duration and cognitive penetrability
}

\author{
Carrie Figdor \\ (Forthcoming in Brogaard and Gatzia, eds., The Rational Roles of \\ Perceptual Experience: Beyond Vision (Oxford)) \\ Please contact the author for use or citation of material in this \\ draft.
}

\begin{abstract}
This paper considers the cognitive penetrability of our experiences of the durations of everyday events. I defend an account of subjective duration based in contemporary psychological and neurobiological research. I show its philosophical adequacy by demonstrating its utility in explaining the phenomenology of duration experiences. I then consider whether cognitive penetrability is a problem for these experiences. I argue that, to the contrary, the problem presupposes a relationship between perception and belief that duration perceptions and beliefs do not exhibit. Instead, the assignment of epistemic features to particular processing stages appears to answer to pragmatic needs, not psychological facts.
\end{abstract}




\section{Introduction}

While "everyday life consists of picnics and meetings just as it consists of chairs and birds" (Zacks et al. 2007: 23), events have been given relatively short shrift in perception research. Similarly, problems in the epistemology of perception are framed almost exclusively in terms of objects and their properties, in particular those perceived visually. This paper extends the philosophical debates to include the epistemology of event perception. The main phenomenologically accessible difference between object and event perception is the experience of temporal information in the latter. Although temporal experience includes temporal order, simultaneity, and duration, I focus on our experiences of duration, and consider mainly their relation to the problem of cognitive penetrability. ${ }^{1}$ Is the perception of the temporal unfolding of events cognitively penetrated? If so, is this cognitive penetration epistemically pernicious?

In Sec. 1, I present the problem of the experience of duration from a philosophical perspective. I then offer an empiricallybased account of how we perceive durations. I focus on perceptions of everyday events occurring within timescales of seconds to minutes to hours. This is the range of interval timing, which lies between millisecond timing (involved in speech perception and motor control) and circadian timing (regulating the 24-hour sleep-wake cycle). On this account, such experiences are a consequence of event segmentation and interval timing processes. This account provides insight into the philosophical problem of duration, and shows how experiences of the now are derived from experiences of durations.

In Sec. 2, I consider the problem of cognitive penetrability and its implications for the justification of perceptual beliefs about duration. I argue that duration experiences and beliefs can't

\footnotetext{
${ }^{1}$ I will use "perception" throughout to mean "conscious perception", which is standard usage. An exception is Raftopoulos 2000, who uses "perception" (in the case of vision) as processing up to Marr's 2.5D sketch and "observation" for perceptual experience.
} 
be divided into the stages required to formulate the problem. In interval processing, stored temporal information is combined with nonstored nontemporal information to produce both duration experiences and beliefs. The problem of cognitive penetrability requires belief to influence perception, whereas interval processing yields both belief and perception.

An independent philosophical debate regarding temporal experience, which I will set aside, concerns the metaphysics of time. This is the debate between the A- and B- theories of time. They agree that time exists, but differ on the nature of tense. An Atheorist holds that the present (the "now" or "specious present") is ontologically special, and that the past and future do not exist. The B-theorist holds that past, present, and future are all equally real, and that the present is not ontologically special (although it may be special for psychological reasons; of course, it can also be psychologically special for A-theorists). Temporal experiences can be veridical if there is a past, present, and future that we track, whether the A-theory or B-theory of time is correct, although not if time itself is an illusion. So aside from global skepticism about the reality of time, this debate does not affect the question of whether event percepts justify event beliefs. ${ }^{2}$

For the same reason, I also set aside background issues in the metaphysics of events. While events seem to be perennially suspect from an ontological perspective, arguments from parsimony for denying ontological commitment to events cut both ways. Four-dimensionalists hold that objects are very slow events, and process metaphysicians hold that processes are fundamental. Everyday perception is consistent with a four-dimensionalist metaphysics as well as one that downgrades events to properties of or relations between objects. The prevailing contemporary view is that events are particulars, not universals (Casati and Var-

${ }^{2}$ Callender (2010) summarizes the contemporary debate in physics about the existence of time as a fundamental physical magnitude. This debate leaves open its existence as an emergent magnitude, in relation to which temporal experiences can be veridical or illusory. I will assume time is (at least) an emergent property. 
zi 2005). Even so, there are sharply different ways of individuating them (Quine 1985; Davidson 1967; Kim 1973). I will assume the coarse-grained view of individuation adopted explicitly or implicitly in the empirical literature on event perception. A coarsegrained view entails that the same token event might have changed in certain ways, including in its duration, and remained the same event. This is plausible because there are modalityspecific limits below which we cannot distinguish distinct events in that sense modality, and because we tend to individuate longer events by goals and intentions rather than durations.

\section{Section 1. The philosophy, psychology, and neuroscience of ex-} periences of duration

1.1. The experience of duration in philosophy. Kelly (2005: 210 ) isolates the experience of concern here in terms of what he calls the Puzzle of Temporal Experience: "How is it possible for us to have experiences as of continuous, dynamic, temporally structured, unified events given that we start with (what at least seems to be) a sequence of independent and static snapshots of the world at a time?" I call this the puzzle of duration, given that the phrase "temporal experience" also includes experiences of simultaneity and temporal order. ${ }^{3}$ While it is not obvious phenomenologically that we do start with what seems to be a sequence of snapshots, the fundamental question is how we come to experience events as occurring through time at all.

A vivid illustration of this type of experience involves hearing a soprano at the opera. The soprano hits a high C, and holds it, and holds it, and holds it, and holds it ... and at some point, as Kelly (op.cit). 208) puts it, what we hear "no longer seems to be

\footnotetext{
${ }^{3}$ These experiences generate distinct puzzles and are also targets of empirical research. The Time-Stamp Problem is about when in temporal order we experience something as having occurred, and the Simultaneity Problem is about which events we experience as simultaneous (Kelly op.cit.). He also distinguishes the puzzle of duration from the hard problem of consciousness, which he sets aside. In contrast, Merino-Rajme (2014) considers the hard problem of consciousness extended to certain temporal experiences.
} 
limited to the pitch, timbre, loudness, and other strictly audible qualities of the note. You seem in addition to experience, even to hear, something about its temporal extent. ... one is tempted to say ... that the note now sounds as though it has been going on for a very long time." A minimally apt description of this experience is that we do experience it as having an unusually long duration, which entails that we have an experience of its duration. This type of experience is also present when we consider ordinary events, such as brewing coffee or checking out items at the grocery store. Unlike a passive acoustic experience of hearing a single held note, many of these events have temporal subparts, involve multiple objects, include our active participation, and integrate multimodal sensory inputs, each with proprietary temporal-perceptual properties. Simple or complex, the experience of duration marks a psychologically important difference between perceptual experiences of ordinary events and ordinary objects. How do we explain it?

Kelly considers two philosophical theories of how we experience duration, and argues that both are inadequate. These are what he calls the Specious Present Theory and the Retention Theory (Dainton 2017 calls them the extensional and retentional theories). On the Specious Present Theory, which he associates with William James, the present of which we are directly aware may be considered to be somewhat extended in time, like a saddle-back. We do not perceive a static snapshot, but instead a temporally extended duration that includes what has recently occurred and what is about to occur. ${ }^{4}$ This proposal requires either that we have direct perceptual access to the recent past and future moments close to the present, or else that we are directly aware of the duration. Neither choice makes sense, Kelly argues: we don't have direct perceptual awareness of what has recently occurred or what is about to occur, while the "default position" on direct awareness

\footnotetext{
${ }^{4}$ Poppel (2004) has argued that our neurocognitive machinery is such that the sensed now is in the range of 3 seconds. Kelly also takes note of Poppel's claim.
} 
seems to be that we are directly aware of what is present now, not of duration.

The Retention Theory, which Kelly associates with Husserl, holds that perception presents us with snapshots, but that these momentary snapshots of which we are directly aware in perception are augmented by states of retention and protension. These are not memories and anticipations, but sui generis intentional acts. Kelly argues that this theory merely provides labels for what must be explained. For example, retention is defined as a way of being directed towards objects and events as just-having-been. It is neither instantaneous remembering (such as when one suddenly remembers having left one's keys in the kitchen), nor entertaining a memory (in the way one can relive now a past event). What we need is an explanation of what it is to experience something as just-having-been that goes beyond saying it is a phenomenon involved in the experience of duration. Kelly does not elaborate on the nature of protension, but presumably a similar problem would arise for that state as well.

Kelly does not try to solve the puzzle of duration, but hopes to have shown that there is an interesting problem to be addressed. In what follows, I will elaborate an account of duration based in contemporary science and articulate its solution to the puzzle. With the account's prima facie empirical and philosophical validity thus established, our experiences of duration so understood will be examined in the discussion of the cognitive penetrability of duration perception in Part 2.

1.2. The psychology and neuroscience of event perception. Event segmentation is analogous to, and as basic as, object individuation: even infants are able to parse evolving scenes into discrete segments (Wynn 1996). This is a fruitful perspective from which to understand duration perception because durations are a matter of interval timing, and event segmentation yields the intervals perceived as durations. Interval timing is the midrange of timing involved in conscious time estimation, cognitive processing (such as decision-making) and behavior (such as foraging) 
(Buhusi and Meck 2005: Fig. 1). Many ordinary events, including those targeted in event segmentation research, fall in this range.

A leading contemporary theory of how we segment continuous activity to generate perceptions of discrete events is Event Segmentation Theory (Zacks and Tversky 2001; Zacks et al. 2007; Kurby and Zacks 2007; Zacks, Tversky, and Iyer 2001; Reynolds, Zacks, and Braver 2007; Tversky, Zacks, and Hard 2008; Shipley and Zacks 2008; for a predecessor, see Newtson, Engquist, and Bois 1977). The targets of EST are perceptual experiences of Quinean events, whether dynamic (events) or concrete (objects) (Zacks and Tversky 2001: 5). ${ }^{5}$ An event is defined as a segment of time at a given location that is perceived by an observer to have a beginning and an end. This definition, while not exhaustive, includes events that involve goal-directed human activity and have durations from seconds to tens of minutes (Zacks and Tversky 2001: 5).

In EST, event segmentation is a side effect of our use of prediction for perception. It is thus is a specific case of the predictive processing or predictive coding framework of overall brain function (Kurby and Zacks 2007: 73, Friston and Stephan 2007; Clark 2013; Hohwy 2014). ${ }^{6}$ It is considered a spontaneous, ongoing process that does not require conscious attention and occurs at various timescales simultaneously. The model posits event models and event schemata. Event models are multimodal, actively maintained representations in working memory of "what is happening now" (Zacks et al. 2007: 7). Their content is influenced by event schemata, which are semantic memory representations

\footnotetext{
${ }^{5}$ This perspective coheres with Kelly's position that adequate answers to the puzzle of duration should not fundamentally distinguish between object and event perception, even though their relation to interval timing and perceived duration differs phenomenally. I set aside here the question of how interval timing is related to object perception.

${ }^{6}$ While publications elaborating EST do not cite major advocates of the predictive processing framework, the conceptual relationship is obvious and sometimes explicit (e.g. Radvansky and Zacks 2017: 133).
} 
of information from previously encountered events and are encoded in permanent synaptic changes.

The mechanism of EST is the detection of variations in the incoming sensory stream and comparison of them to values predicted by event models. Transient increases in prediction error based on comparison between actual and predicted sensory input at a given timescale triggers updating of the relevant event model, after which another period of stability begins. This increased processing is perceived as the subjective experience that a new event has begun, while periods of stability are perceived as ongoing events (Kurby and Zacks op.cit.: 72). We can selectively attend to particular timescales in response to instruction, but also spontaneously segment at finer grains when there is less predictability and we seek more information to understand what is going on. The account implicitly distinguishes experiences of duration from those of succession: succession implies at least two events, while durations are of one. Philosophical accounts of duration sometimes conflate succession and duration as one phenomenon (Phillips 2014: 140). ${ }^{7}$

Zacks et al. (2007: 4) illustrate EST with the example of a man scraping plates in the course of washing dishes. The whole plate-scraping segment of the dish-washing event is predictable until the last plate is scraped, when the goal of scraping the plates would no longer have predictive value and updating mechanisms would kick in. At a more fine-grained timescale, each platescraping activity will generate a small predictive error when that plate has been scraped, and this error will correspond to a boundary between each individual plate-scraping. It follows that prediction errors will be relative to timescales, such that variations in input that count as prediction errors at one timescale (the start of a particular plate-scraping) fall within expected or predicted lim-

\footnotetext{
${ }^{7}$ It also may conflict with Phillips' (2014) "naïve" account of the relation between objective durations and subjective experiences of duration, in which the latter "inherit" the "temporal structure" of the former. It depends on how "inheritance" is elaborated.
} 
its at another (the whole dishwashing activity). The boundaries of each dish-scraping are prediction errors relative to the timescale of the event model for plate-scraping, but are within predicted values at the timescale of the dish-washing event model in which the dish-scrapings occur. Many everyday events will exhibit this complexity: they have temporal segments (entailing part-whole relations) and temporal abstraction relations across timescales and levels of generality (entailing hierarchical relations). ${ }^{8}$

While event schemata are explicitly thought to include such items as goals and statistical information about paths and motions of objects, they also presumably contain statistical information about how long types of events generally take. In classical conditioning, after learning the subject is able to predict the duration between stimulus onset and reward presentation and will begin to exhibit anticipatory behavior when the reward is nigh. But top-down expectations of duration can also influence the quality of ongoing experiences of duration without inducing prediction errors and thus segmentation. For example, we may know from experience that a typical episode of checking out groceries takes a few minutes. An event model for a token of this event may reliably predict a temporal range within which the event should end. With this range and goal set, there is no need to allocate attention to its duration if it is taking about as long as expected. When the duration extends to the far end or beyond the approximate range, our experience may qualitatively change: we suddenly become aware that we are in a very slow checkout line. The qualitative difference may be felt as a change of affect (e.g. impatience) that is assigned to the duration rather than some other aspect. But there is no segmentation: the duration that comes to seem too long is the duration of the same event. ${ }^{9}$

\footnotetext{
${ }^{8}$ Thanks to Andy Clark for raising the need for this clarification.

${ }^{9}$ Animals likely have similar qualitative differences: your dog experiences a duration between when you walk in the door and when he is fed, but this experience (and not just his increasingly frantic behavior) can change in quality if you are slower than usual in feeding him.
} 
These changes in our experiences of ongoing durations make vivid the fact that while EST tells us when and why event boundaries are perceived, it says little about how event durations are perceived. It says only that they may be perceived during periods of processing stability. Nevertheless, since event segmentation yields intervals, EST is ipso facto a theory of what activates or triggers interval timing and thus of what makes experiences of durations possible. We can therefore expect to gain further insight into experiences of duration by augmenting EST with what we know about our internal interval timing mechanism, sometimes known as the stopwatch. While the circadian clock has been localized in the suprachiasmatic nucleus and millisecond timing in the cerebellum, the stopwatch involves thalamo-cortico-striatal circuitry, including the basal ganglia, supplementary motor area, prefrontal cortex, and posterior parietal cortex (Allman 2014: 746 and Table 1; Buhusi and Meck 2005; Grondin 2010). ${ }^{10}$ After two preliminary remarks, in the rest of this subsection I briefly summarize main themes from the literature on interval timing, combine EST and the leading theory of interval timing, and indicate how the combined account makes contact with epistemic concerns.

The first, minor, remark is that much psychological and neurobiological research on interval timing has been directed at unimodal events with timescales of a few hundred milliseconds to a few seconds (Matthews and Meck 2016, Grondin 2010). Scalingup assumptions are implied when results are extended to longer intervals and more complex events within the midrange. ${ }^{11}$

${ }^{10}$ These timing mechanisms interact: for example, older adults make more accurate duration estimates in the morning, young adults in the evening (Allman et al. op.cit: 758).

${ }^{11}$ For example, Hommel et al. 2001 propose a theory of common coding for event perception and action planning. While their focus is simple, brief events like key-pressing tasks, Zacks (2001: 910) and Hommel et al. (op.cit.: 914) agree that a modified version of their basic framework should apply to longer events (e.g., making coffee). 
The second, major, remark is related to the distinction in research protocols between those eliciting prospective timing judgments and those eliciting retrospective timing judgments (Wearden 2005). In prospective timing experiments, human or animal subjects are told (or trained) in advance that an interval estimate (provided verbally or behaviorally) will be sought (e.g. "Hold down the button for 1 second"). In retrospective timing experiments, human subjects are asked unexpected questions about intervals (e.g. "how much time has passed since you started reading this paragraph?"). The targets of these estimates are considered distinct: prospective judgments are reports of episodes of interval timing, and retrospective judgments are outputs of general cognitive mechanisms not specifically related to time. For illustration, in Kelly's case of the soprano presumably we could have made a prospective judgment of the note's duration had we been asked to do so. In contrast, cases where (e.g.) after 25 minutes of a boring movie you say, "It feels like the movie has been going on for an hour" (Merino-Rajme 2014), we are giving retrospective judgements (and arguably in this case we are really reporting an affective response). The internal clock theory discussed below is about the mechanisms of interval timing whose experienced outputs are reported in prospective estimates. The importance of this point will become clear in Section 2.

The stopwatch temporally integrates multimodal sensory signals that arrive at different speeds and are processed at different speeds by sensory organs, compensating for these differences so that we can track discrete events and objects. The dominant information-processing hypothesis of interval timing is the pacemaker-accumulator or PA model (Treisman 1963; Wearden 2005; Allman et al. 2014; Buhusi and Meck 2005; Eagleman et al. 2005; Grondin 2010; Block and Grondin 2014). The model implements the Scalar Expectancy Theory or SET (Gibbon 1977; Gibbon, Church, and Meck 1984; Buhusi and Meck 2005) in that it was formulated in part to explain the scalar property of interval estimates. The scalar property is the fact that these estimates are more variable relative to the mean as the length of the interval 
grows, by a fixed proportion that follows Weber's law. The errors are larger for larger intervals, although not relatively larger than errors for shorter intervals (Wearden 2005). Gibbon et al. (1997: 170) liken this feature of subjective timing to a rubber ruler that can be stretched to measure any arbitrary target interval, but where interval estimation error increases proportionally as the ruler is stretched.

Note that the scalar property also applies to neural responses (Buhusi and Meck op.cit.: 756, Fig. 2). So while interval estimates by human and animal subjects are reliably correlated with and interpreted as reports of experienced intervals, the psychological concept of subjective interval timing includes more than just experienced intervals. This coheres with EST's view that event segmentation proceeds nonconsciously as well as consciously. Also, Block and Grondin (2014: 1) question whether the scalar property should be taken for granted as an explanandum of an adequate theory of interval timing, given that it is directly a feature of time estimation rather than of time perception (which in their usage refers to what interval timing mechanisms do, whether subjects perceive the intervals or not). However, by seeking to explain both the PA model affirms a tight relationship between experienced and estimated intervals in prospective timing. ${ }^{12}$

The PA model posits a pulse counter (a clock stage), a reference memory (a memory stage) and a comparator (a decision stage), each with its own forms of variance that can contribute to inaccurate perceptions of duration. At the onset of an event, a pacemaker emits pulses (neural spikes) that are gated into an accumulator by attention. The pulse tally is transferred to working memory for comparison to an interval value for that type of event

\footnotetext{
${ }^{12}$ A minority view of interval timing (e.g., Staddon and Higa 2006) is that it is not performed by a specialized mechanism but by basic memory and learning processes. Buhusi and Meck (2005: 763) hold that interval timing is a specialized mechanism, but that it shares neural circuits with non-temporal processing. (For comparison: the circadian clock is considered a specialized mechanism, and it has dedicated neural wetware.)
} 
stored in reference memory. (In prospective timing experiments, this is often an interval just presented to the subject.) This step is also described in terms of the transfer of the pulse count directly to reference memory for comparison to the stored reference interval (Allman et al. op.cit: 750). Either way, when these values are close enough, a decision rule determines an interval estimate and appropriate response. ${ }^{13}$

EST and the PA model can be integrated in part in the following way. If EST is correct, working memory contains an active event model whose predictive processing of incoming stimuli triggers the first stage of the PA model; presumably nonattentional mechanisms can also do the gating in this stage. Working memory also comes to contain the pulse tally received from the accumulator in the second stage of interval processing. Importantly, there must be coordination between the event model that generates prediction errors (event boundaries) and the reference interval to which the accumulated pulse count that begins at a given boundary is compared. A simple hypothesis is that the reference interval is part of the event model, although other relations are possible. However coordination occurs, event schemata presumably influence duration perception as well as boundary perception by helping determine which reference interval is relevant. This may included in determining which event model is active. For example, the event-model for dish-scraping includes information about how long this type of event normally takes, and the interval that is triggered at the start of the dish-scraping is compared to this reference interval. Given that perceived boundaries and subsequent perceived intervals together constitute perceived durations, it follows that both constituents of duration per-

\footnotetext{
${ }^{13}$ The coincidence-detection (or striatal beat frequency, SBF) model of interval timing holds that patterns of spikes of cortical neurons are continuously compared by striatal spiny neurons with a reference pattern. It may be that coincidence-detection and pulse counting are just two ways of comparing a stimulus-dependent quantity to a reference interval (Buhusi and Meck 2005: 763). If so, the SBF model is of the neurobiological implementation of the first step of the PA model, not an alternative theory.
} 
ception are subject to top-down influences. I return to this point in Part 2.

In addition, the fact that we make proportionally larger errors when estimating larger intervals may help explain why, as events increase in temporal length, they are more characterized by goals, plans, or intentions rather than physical features such as motion (Zacks and Tversky 2001), and why beginnings loom larger than endings (Teigen et al. 2017). We compensate for the stopwatch's loss of precision at longer intervals by using more reliable alternative methods to fix when longer events are over. A non-elite marathon runner may have a vague idea of her time when she crosses the finish line, but she knows exactly when she has finished. We can of course get better at time estimation. Elite marathon runners are duration experts analogous to the way sommeliers are wine experts: they can accurately distinguish durations of 2 hours from 2 hours and 5 minutes. In any case, there is no reason to think estimates of durations of everyday events must be precise to be accurate. Accuracy often only requires falling within a range. ${ }^{14}$

The neurobiology of interval timing is being actively investigated. A biological basis for the stopwatch was initially inferred from the fact that higher bodily temperatures in fever resulted in altered subjective judgments of passing time (Wearden 2005: 9 Fig. 1). Within the PA model, heating implies more clock ticks per objective interval and more quickly accumulating the number of pulses in the reference interval associated with the event being timed. The result is experiencing and estimating intervals as longer than they really are: when instructed to count out a minute, we may count out (what feels like) 60 seconds in less than $45 \mathrm{sec}$ onds. Perceptions of durations are also affected by a variety of

\footnotetext{
${ }^{14}$ Our baseline accuracy for judging intervals differs across modalities (e.g. audition is generally more accurate than vision (Allman et al.: 746)). We also tend to perceive events as occurring closer in time then they are when the events are an action we did and its effect (Ebert and Wegner 2010; Andersen 2013), although measured effects are in the millisecond range. Interval timing ability also varies across subjects.
} 
sensory, psychological, and physiological factors, including attention, arousal, memory, affect, psychiatric disorders, and drugs affecting neural and neurotransmitter activity (Matthews and Meck 2016; Allman et al. 2013; Droit-Volet and Meck 2007; Buhusi and Meck 2005; Grondin 2010; Cheng et al. 2006; Terhune et al. 2014).

In terms of the PA model, these factors can change the pulse rate (clock speed), the working memory representation of the reference interval, or the baud rate at which accumulated pulses are transferred into working memory. For example, dopaminergic agonists (e.g. methamphetamine) increase clock speed while antagonists (e.g. haloperidol) decrease it (Allman et al. op.cit.: 749; Buhusi and Meck op.cit.: 757), and cholinergic antagonists (e.g. atropine) affect reference memory in that the criterion interval a subject uses is longer than the intervals that were presented in training. Given the PA model, at least some of these influences can explain why car accidents may be experienced as occurring in slow motion. Due to sharp, rapid increases in attention, affect, and stress, external time is experienced as abruptly slowing down because the internal clock rate has rapidly accelerated without a corresponding shift in the reference interval for normal driving that was active. Yet while we may perceive everything in slow motion, our millisecond timing mechanisms can still control our motor responses automatically. ${ }^{15}$

1.3. The Philosophical Adequacy of the Account. The grounding of duration perception in perception of the now appears to be a common background assumption of the Specious Present and Retention Views. Perception of now is the basic item of temporal perception, whether it is instantaneous or somewhat stretched. However, if the above account is on the right track, we

${ }^{15}$ Arstila (2012) suggests that norepinephrine is likely to play an important role as well, in particular for attention shifts. On a higher-level account (Tse et al. 2004), the added attention may result in fewer temporal cues being missed ("missed temporal cues" interpretation) or the counting of more units or pulses by the stopwatch ("attentional boost" interpretation), or both. The account in the text is not intended to be complete. 
do not perceive duration as a number of stitched-together nows (or overlapping brief successive experiences: Dainton 2014). Perceiving durations is perceiving boundaries plus intervals - i.e. maxima of processing changes that trigger the stopwatch and periods of processing stability between these boundaries while the stopwatch ticks away. The experienced now is just an ordinary, smallish experienced duration - ordinary and smallish because we are easily able to perceive ordinary events with durations far briefer than 3 seconds. "Now" is a vague term that picks out these intervals. There is nothing left to explain of the now once we explain duration in terms of event segmentation and interval timing. Note that this is not an account of simultaneity or temporal order of successive events, which are distinct explananda.

An imperfect metaphor for our experiences of duration is many reels of film with irregular frames (events at varying timescales with varying durations) running through a movie projector at once. The now is what is in the projector's light, whether this includes at least one consciously accessible boundary or not. In these terms, the traditional debate of extensional versus retentional views of experiences of duration concerns whether what is in the projector's light is extended or momentary (see, e.g., Dainton 2014: 103 Fig. 6.2). The empirically-based account does not ignore philosophical concerns to explain how conscious experience seems (continuous, unified, etc.); it interprets phenomenological descriptions of experiences of duration and the now in the light of what we know about interval processing.

But Kelly might insist: how is it that we both perceive the soprano as singing the note now and yet perceive that this acoustic event has been going on for a long time? I suggest that this description picks out temporal and nontemporal elements of our phenomenologically accessible experience. The nowness of the event's duration is the accessible segment of an ongoing duration. A second aspect of our experience is generated by enhanced attention to this duration, which is prolonged beyond expectation on the assumption that the aria is not familiar. (If we are opera experts, we will not experience the note as too long - not unless it 
violates the composer's actual notation.) This is worthy of additional attentional resources in the light of our limited knowledge. Kelly describes this sudden change in our experience of the note as perceiving that it has been going on for a long time. But we are not reporting experiencing the soprano's singing as slower or faster. Those changes would be reported as changes in experienced duration. Our stopwatch is clicking away at its normal speed. Instead, we are reporting another aspect of our experience - an experience of astonishment directed at its duration, rather than, say, its pitch. This is a phenomenological change in our experience of its duration, but it is not due to a change in timing, and so a report of it is not a report of our experience of timing. The account of duration helps us distinguish features of duration experiences that phenomenological description does not.

Kelly also mentions that the received view of our experience of the now is that it is direct. For some, perception is direct in those cases when it can be appropriately contrasted with cases where inference (or some other cognitive operation) is added to perception. For example, I perceive my car by looking at it, but may use inference to recognize it as my car if it has been totaled (Gallagher 2008). By the account given above, experience of the now is direct by this definition. On the other hand, for others perception is direct if percepts contain exclusively sensory information, independently of any other conscious source of information, such as belief or memory (Chuard 2011). By the account given above, experience of the now is not direct by this definition. Chuard also asks (2011: 4) whether temporal properties and relations are among the "strictly speaking perceptually accessible" properties - that is, if they are perceptible "in the same way, that is, as shapes, colours, and spatial relations" are perceptible. They aren't, but so what? Appeals to directness or strict perceptual accessibility do not seem to isolate important features of temporal experiences.

\section{Section 2. The epistemology of event perception}


2.1. Perception, Justification, and Cognitive Penetration. The main question in the epistemology of perception is whether beliefs about the external world are justified by the perceptual experiences they are occasioned by (Siegel and Silins 2015). ${ }^{16}$ Three main sources of doubt that they are so justified include the underdetermination or ambiguity of perceptual content by sensory stimuli (underdetermination), the biasing influence of cognitive states (cognitive penetrability), and the possibility of global illusion (skepticism). Underdetermination and cognitive penetrability can overlap when cognitive states (or sources of information considered cognitive) disambiguate the sensory input. Philosophers have discussed these concerns primarily in relation to visual perception of and beliefs about objects and their features. Here I extend the discussion of cognitive penetrability to events and their durations, setting aside the problems of skepticism (or illusion) and underdetermination. ${ }^{17}$ Also, in contemporary discussions, experiences are taken to have externally directed contents, and perceivers need not believe that things are as experiences represent them to be (Siegel and Silins op.cit.: 782). Just so, our experiences of duration are about external events, and we can have experiences of durations that we do not believe are veridical

\footnotetext{
${ }^{16}$ Siegel and Silins distinguish between propositional justification, when an experience provides reason to believe something whether one comes to believe it or not, and doxastic justification, in which a belief is based on experience more specifically, a belief is doxastically justified iff it is rationally formed, adjusted, or maintained on the basis of experience (Siegel and Silins op.cit.: 784). In another use of "doxastic", doxastic states are those accessible to consciousness and inferentially integrated (Macpherson 2017: 11).

${ }^{17}$ The ambiguity of event perception is revealed by the McGurk effect and the sliding/bouncing effect (Sekuler et al. 1997; Watanabe and Shimojo 2005). Experiments inducing illusory duration judgments suggest that the stopwatch is constantly being calibrated to the external world (Eagleman et al. 2005). Moreover, there must be coordination between the timing and durations of our own movements and the movements or motions of other entities for the purpose of adaptive action (Hecht 2000: 18; Kurby and Zacks op.cit.: 78; Hommel et al. 2001: 877).
} 
(e.g. the illusion of time passing in slow motion during a car accident).

To a first approximation, cognitive penetration of perception occurs when beliefs, expectations, desires, hopes, goals and other cognitive states intervene in the perceptual process so as to affect the nature of the perceptual experience (Stokes 2013; Silins 2016: 24; Vance 2015; Siegel 2012: 205-206). This difference in the nature of the experience is usually spelled out at least in part in terms of a difference in the experience's content. Macpherson (2012: 29) holds that a perception is not cognitively penetrable if it is not possible for any two perceivers (or the same perceiver at different times) to have experiences with distinct content or character when one holds fixed the object or event of perception, the perceptual conditions (e.g. lighting), the spatial attention of the subject, and the conditions of the sensory organs(s). We may add temporal attention to the list (e.g., attention to the soprano's singing). Macpherson (2017) further specifies that in cognitive penetration there must be a semantic and causal link at each step from the belief (or other cognitive state) to the perception. ${ }^{18}$

Cognitive penetrability of perception is an epistemic problem in the light of traditional views of how perception works and how it is supposed to provide epistemic justification. The metaphysical presupposition is the existence of a theoretically important perception/cognition distinction. The epistemological presupposition is the idea that percepts must be linked to the external world without mediation by cognitive resources to have justificatory power. They should be "untainted" by prior assumptions made by the subject or a subpersonal part of her perceptual system (Vance 2015: 643). As Silins (2016) puts it, your experience "reflects what is before you and does not reflect your own mind. Given that your experience is not influenced in any way by your theories or expectations, it is thereby in an optimal position

\footnotetext{
${ }^{18}$ Macpherson (2017) also distinguishes between cognitive penetration of early vision (e.g. Pylyshyn 1999: 343) and of perceptual experience; the latter is of concern here.
} 
to confirm or disconfirm hypotheses about the world." These two presuppositions ground the idea "that the content of the perception underwrites that of the belief it justifies, in the sense that it logically implies it, or makes it probable, or maybe just in the sense that an inference from the perception to the belief would, in the present context, be reliable" (Heck 2000: 499). Cognitively penetrated perceptions lack the content independence required for them to provide rational justification for belief.

Siegel $(2012,2017)$ provides a compelling example of the worrisome circularity: Jill believes (without good grounds) that Jack is angry at her, she sees his face as being angry because of this belief, and she takes this perception at face value to justify her belief that he is angry. He does, after all, appear angry to her. In this way, the penetrating cognitive states are "stacking the tribunal of experience" in their favor: the experience does not provide an independent reason for your belief (but see Lyons 2011). Of course, we also know that learning can improve your perceptual experiences and lead to better beliefs than one might otherwise have. A tree expert knows what an elm looks like and will perceive elms more quickly and accurately, and these perceptions will redound to the credit of her prior elm beliefs. If these are cases of cognitively penetration, then cognitive penetration is not necessarily pernicious. Regardless, it seems clear that a problem can arise in some cases.

2.2. Experiences of Duration and Cognitive Penetrability. On the EST-based account, experiences of duration are made possible by the triggering of the stopwatch by prediction errors generated in the course of processing nontemporal sensory inputs. EST builds top-down influence into event segmentation, and thus potentially into experiences of event boundaries, via the influence on event models of prior knowledge contained in event schemata. I elaborated this account in plausible ways. Schemata presumably include statistical information about previously experienced event durations. The influence of this information can be expected to extend to experiences of event duration, given the need to coordinate segmentation and interval timing processes. Coordination 
would be achieved if event models simply contained previously learned reference intervals. In short, the proposed account of event perception appears to raise worries of cognitive penetrability about experiences of duration.

One reason not to worry stems from the fact that the presupposed divide between perception and cognition is undermined by scientific advances. Without that distinction, cognitive penetrability may be impossible given how it is defined (Macpherson 2017). Even if it is not ruled out by definition, in predictive processing or broadly Bayesian accounts of overall neural function, the outputs are our best models of the world. What is fed forward from the sensory stream is a prediction error (if any) generated by a difference between the expected sensory stimulation and what is detected. As Shea (2015: 76-77) puts it, in predictive processing there is no "cascade of illusory justification" bubbling up from below, just the nudging of pre-existing models towards better predictions using prediction errors. The account of duration perception defended above does not quite escape the worry for this reason, because while EST is a predictive processing model the PA model is not. But it could easily be revised into one - for example, by interpreting the reference interval as a predicted value of an active event model. In this case, the worry would be alleviated for duration perception as it is for any predictive coding model.

However, Shea suggests that an updated reformulation of the perception/cognition distinction that might enable us to pose some of the questions raised in the cognitive penetrability debate. It has turned out that many input-driven processes important in cognition turn out to straddle the traditional divide. They have features that usually belong to systems in the cognitive category. For example, while they are input driven they have an "amodal" phenomenology in that they "take as input whichever kinds of sensory inputs are relevant in the circumstances" (Shea 2015: 85). Among these hybrid systems are the system for representing one's spatial position, language processing, and Carey's systems of 
core cognition, such as numerosity for representing quantities of items or perception of agency.

Our interval timekeeping mechanisms also belong in the hybrid class. The stopwatch is amodal in that it is driven by nontemporal inputs in the sensory modalities that are relevant in the circumstances. It also operates at many timescales, some fast and mandatory, others slower and under cognitive control. Note that our timing mechanisms are not on a par in this respect. Millisecond and circadian timing are automatic and largely not consciously controlled. Circadian timing in particular satisfies many if not all of Fodor's (1983) original criteria for modularity - it is automatic, has dedicated neural wetware, and is to some degree encapsulated in that its operation is impervious to belief about the actual time right after a long flight. Interval timing operates like a central system by Fodor's standards. It is subserved by neural circuitry spanning many cortical and subcortical regions of the brain. Influences on the activity of the stopwatch that affect our experiences of duration can come from below (e.g. drug-induced or externally stimulated variation in neurotransmitter activity, affecting the pulse rate of the stopwatch) and above (e.g., memory defects and emotional states, affecting the reference interval). These effects occur in normal and abnormal experienced intervals.

Shea suggests that we can at least characterize how much of a mechanism's operation is driven by current input and how much by top-down information. Not all pre-existing information is top-down. For example, a system can contain prior information in the form of expectations, with the latter understood as dispositions to transition from one representation or another in certain ways. These do not count as top-down effects, but are the effect of learning and may be beneficial. Top-down information is occurrent and explicitly represented, such that it can act as input to many different systems. More precisely, a top-down influence is "a representationally mediated effect of an explicit representation $R$ on a psychological process, where $\mathrm{R}$ is not computed more directly than the representational influence of current sensory inputs 
on the process" (Shea op.cit.: 81)..$^{19}$ This makes top-down influence a matter of degree. The surviving epistemic question would be whether the output of one system A is suitable for belief formation (or other subsequent processing) in another system $B$ if B's representations have affected A's processing. On this view, independence from top-down influence is not needed for epistemic suitability. Top-down information can influence a psychological process whose outputs reinforce that information without pernicious circularity.

Shea's proposal has the virtues of updating the debate to take into account the actual complexity of much processing, and to allow that the epistemic implications of top-down influence vary from case to case. The traditional presuppositions are gently modified to leave some form of the traditional worry behind. Unfortunately, once we acknowledge the complexity of actual processing, a third presupposition of the traditional debate is left unsupported, and is unsupportable.

Perceptual belief is traditionally understood as being formed on the basis of experience: the beliefs are occasioned by the perceptions. We see a red tomato and form the belief that it is red. Structurally, there are two processes connected by a route for information flow. Both the original perception/cognition distinction and Shea's top-down/bottom-up distinction embed this routing assumption in which information flows from process $A$ to process $B$, and maybe from $B$ back to $A$. (There is no circularity without a routing assumption.) This structure presupposes that $A$ and $B$ are (or are the outputs of) distinguishable processes. We have percept-forming processes, and their outputs can be inputs to belief-forming processes. In cognitive penetration, the routing also goes the other way: outputs of belief-forming processes influence percept-forming processes. Predictive coding and Shea's analysis show that these processes cannot be neatly separated. Duration

\footnotetext{
${ }^{19}$ The "directness" requirement is not patently clear (as Shea recognizes) but I
} will not raise any issues here about it. 
processing shows that even Shea's reformulation does not go far enough in taking account of the complexity.

As noted above, interval timing researchers distinguish between prospective and retrospective judgments or estimates of durations. Prospective judgments are of experienced intervals produced by interval timing mechanisms. Their close relation suggests that duration experiences and prospective duration estimates are outputs of the same interval timing mechanism. They are correlated because they have a common cause in the same endogenous process subject to top-down influences among many others. The duration estimates are not formed on the basis of perception, but along side perception. These estimates are distinguished from retrospective judgments, which are outputs of distinct processing mechanisms not specific to time. In short, researchers distinguish duration beliefs that are about the experiences of duration arising from interval timing mechanisms, and duration beliefs that are not occasioned directly by interval timing at all. Of course, we can describe this processing structure in terms that fit the routing structure behind traditional epistemology of perception and the problem of cognitive penetration: just call the stored or top-down information that influences interval timing a belief. But the duration estimate that is the outcome of interval timing mechanisms is not identical to this belief. The perceptual belief (the estimate of the experienced duration) and the stored belief are not the same. So there is no circularity no matter how much top-down information influences interval timing. Duration perception and estimation do not map onto the abstract structure of the problem.

This analysis points to a larger moral. Most philosophers agree that the behavior and states of agents (or persons, in a bland sense) are to be explained in terms of the operations of subpersonal states. But belief and perception are personal-level states when we refer to them for epistemic purposes. The visual system does not perceive, the person does. It is a big and not entirely coherent step to argue from the fact that subpersonal processing explains personal-level behavior to the idea that epistemic 
features attach to subpersonal processing stages. This treats perception and belief as personal and subpersonal at the same time. It might have been harmless to continue to ascribe epistemic features directly to subpersonal states if the processing details had turned out to be as simple as tradition holds. But in a complex information processing system, what is the value of distinguishing particular stages by epistemic features when it's the overall outcome that is epistemically good or bad? Complex systems routinely adjust to compensate for normal variation or change, as well as defect or insult, without any observable behavioral difference. All stages might contribute a little bit to a lousy epistemic outcome. There are simply too many influences, from too many different sources and functional levels, that can spoil the epistemic broth.

Thus, suppose an on-duty police officer on a call, service weapon in hand, perceives a man's arm movement following a pulling-object-out-of-pocket event as the start of a taking-aim event rather than the start of a raising-hands event, and he shoots the man. A taking-aim event reliably has a much briefer duration than a raising-hands event in these circumstances, but which type of event is starting at the perceived boundary is ambiguous. So we assume the officer perceives the start of an event of predictably very brief rather than predictably somewhat longer duration: the underdetermination of the initial motion is resolved in one way rather than another. Suppose it has been resolved by past experience stored in the officer's event schemata for dangerous policing situations, of which this context is a token. There may or may not also have been a detected difference in motion at the millisecond timescale that governs his automatic response.

Something has gone morally awry if the officer shoots an innocent man. What is not at all clear is whether anything has gone epistemically awry that can be pinned on his subpersonal processing. If we suppose the victim is black, one might argue that the officer had antecedent racist beliefs that somehow influenced his perception, perhaps by making it more likely that this context would be classified as dangerous (or more dangerous than otherwise). But racist beliefs need not impinge on the officer's event 
processing for them to play a role in his behavior, nor on the explanatory interests lead us to blame the officer for his response (or else excuse him, if we are also racists). Beliefs can make us insensitive to evidence (Lyons 2011: 301). Much of the epistemic threat blamed on cognitive penetration may instead arise from confirmation bias and other forms of motivated reasoning, not processing details.

Of course, there are times when we can reliably pick out a particular faulty stage in information processing: she believed that the boulder was a big dog, but she wasn't wearing her glasses. But we extend these simple cases far beyond the point of validity. Our penchant for doing so appears to answer to pragmatic concerns for personal responsibility and reasons-giving, not to concerns for getting the processing details right. It hardly seems to matter how subpersonal information processes work: in the case of the officer, via a heightening of fear that increases the probability of trigger-pulling whatever the current perceptual input, or via top-down influence on perceptual processing of the current sensory input from the man's arm movement, or via an implicit expectation built into his activated event models, or via misapplied reference intervals, or via all of the above. What matters epistemically is uncorrected, uncompensated for, or evidentially problematic learning or evidence gathering.

Perhaps we should distinguish an epistemic role we call belief from a subpersonal psychological state of belief that might fill the epistemic role. In any case, once we accept that we cannot divide information-processing into intuitive routes to which subpersonal assignments of epistemic features might be made, the epistemic problem is just that of whether the person would have behaved better if he had better beliefs or perceptions. The accused officer may plead that he felt he was in danger; he may say, as a retrospective judgment of the man's action, that he perceived the man taking aim. Others may try to pin the bad outcome on racism-penetrated perception. Both responses reflect the custom of assigning responsibility to individuals, extended now to their subpersonal processes or outputs, for when something goes 
wrong. Such assignments do not answer to the facts of actual information processing. But we should not think that is their purpose anyway.

\section{Conclusion.}

This paper had two main aims. First, I provided an empirically-based account of our experiences of duration. My account elaborates Event Segmentation Theory with current research on interval timing mechanisms at both the information-processing and neural levels. Along the way I used this account to provide an explanation of changes in quality of duration experiences and illusory durations. I thus demonstrated its prima facie adequacy in terms of taking both empirical research and phenomenology into account. Second, I considered the problem of cognitive penetrability for duration perception. The EST-based account strongly suggests that experiences of duration involve top-down influences. However, I also argued that the structure of interval perception and belief does not map onto the structure required by the epistemological problem. Maybe subpersonal processing stages can be assigned personal-level epistemic roles for the purposes of assigning responsibility and reasons-giving. But we should not confuse the structure of these assignments with the structure of the underlying processing.

\section{$\underline{\text { References }}$}

Allman, M., S. Teki, T. Griffiths, and W. Meck (2014). Properties of the Internal Clock: First- and Second-Order Principles of Subjective Time. Annual Reviews of Psychology 65: 743-771.

Andersen, H. (2013). The representation of time in agency. In A. Bardon and H. Dyke, A Companion to the Philosophy of Time (Wiley-Blackwell): 470-488. 
Anderson, M. (2015). Mining the Brain for a New Taxonomy of the Mind. Philosophy Compass 10 (1): 68-77.

Arstila, V. (2012). Time slows down during accidents. Frontiers in Psychology 3 (196).

http://doi.org/10.3389/psyg.2012.00196.

Block, R. and S. Grondin (2014). Timing and time perception: A selective review and commentary on recent reviews. Frontiers in Psychology 5: 648.

http://doi.org/10.3389/fpsyg.2014.00648

Buhusi, C. and W. Meck (2005). What makes us tick? Functional and neural mechanisms of interval timing. Nature Reviews Neuroscience 6: 755-765.

Callender, C. (2010). Is Time an Illusion? Scientific American 302 (6): 58-65.

Chuard, P. (2011). Temporal experiences and their parts. Philosophers' Imprint 11 (11): 1-28.

Casati, R. and A. Varzi (2008). Event concepts. In T. Shipley and J. Zacks, eds., Understanding Events (Oxford: Oxford University Press): 31-53.

Cheng, R.-K., C. MacDonald, and W. Meck (2006). Differential effects of cocaine and ketamine on time estimation: implications for neurobiological models of interval timing. Pharmacology, Biochemistry, and Behavior 85: 114-122.

Clark, A. (2013). Whatever next? Predictive brains, situated agents, and the future of cognitive science. Behavioral and Brain Sciences 36 (3): 181-204. 
Dainton, B. (2014). The phenomenal continuum. In Lloyd and Arstila, eds. Subjective time: the philosophy, psychology, and neuroscience of temporality (Cambridge, MA: MIT Press): 101-137.

Davidson, D. (1969). The individuation of events. In N. Rescher, ed., Essays in honor of Carl G. Hempel (Dordrecht: Reidel): 216234.

Droit-Volet, S. and W. Meck (2007). How emotions color our perception of time. Trends in Cognitive Sciences 11 (12): 504-513.

Eagleman, D., P. Tse, D. Buonomano, P. Janssen, A. Nobre, and A. Holcombe (2005). Time and the Brain: How subjective time relates to neural time. The Journal of Neuroscience 25 (45): 1036910371.

Eagleman, D. and A. Holcombe (2002). Causality and the perception of time. Trends in Cognitive Sciences 6 (8): 323-325.

Ebert, J. and D. Wegner (2010). Time Warp: Authorship shapes the perceived time of actions and events. Consciousness and Cognition 19 (1): 481-489.

Fodor, J. (1983). The Modularity of Mind. (Cambridge, MA: MIT Press).

Friston, K. and K. Stephan (2007). Free-energy and the brain. Synthese 159: 417-458.

Gallagher, S. (2008). Direct perception in the intersubjective context. Consciousness and Cognition 17: 525-543.

Gibbon, J. (1977). Scalar expectancy theory and Weber's law in animal timing. Psychological Review 84 (3): 279-325. 
Gibbon, J., R. Church and W. Meck (1984). Scalar timing in memory. Annals of the New York Academy of Sciences 423: 52-77.

Gibbon, J., C. Malapani, C. Dale, and C. Gallistel (1997). Towards a neurobiology of temporal cognition: advances and challenges. Current Opinion in Neurobiology 7: 170-184.

Grondin, S. (2010). Timing and time perception: A review of recent behavioral and neuroscience findings and theoretical directions. Attention, Perception, and Psychophysics 72 (3): 561-582.

Hecht, H. (2000). The failings of three event perception theories. Journal for the Theory of Social Behaviour 30 (1): 1-25.

Hohwy, J. (2014). The Predictive Mind. Oxford: Oxford University Press.

Hommel, G., J. Muesseler, G. Aschersleben, W. Prinz (2001). The theory of event coding (TEC): A framework for perception and action planning. Behavioral and Brain Sciences 24 (5): 849-937.

Kelly, S. (2005). The puzzle of temporal experience. In A. Brook and K. Akins, eds., Cognition and the Brain: the philosophy and neuroscience movement (Cambridge: Cambridge University Press): 208-238.

Kim, J. (1973). Causation, nomic subsumption, and the concept of an event. Journal of Philosophy 70: 217-236.

Kononowicz, T. and V. van Wassenhove (2016). In search of oscillatory traces of the internal clock. Frontiers in Psychology 7: 224.

http://doi.org/10.3389/fpsyg.2016.00224

Kurby, C. and J. Zacks (2007). Segmentation in the perception and memory of events. Trends in Cognitive Sciences 12 (2): 72-79. 
Le Poidevin, R. (2015). The experience and perception of time. In E. Zalta, ed., Stanford Encyclopedia of Philosophy (Summer 2015 edition):

http://plato.stanford.edu/archives/sum2015/entries/tim e-experience

Lloyd, D. and V. Arstila (2014). Subjective time: the philosophy, psychology, and neuroscience of temporality. Cambridge, MA: MIT Press.

Lupyan, G. (2015). Cognitive penetrability of perception in the age of prediction: Predictive systems are penetrable systems. Review of Philosophy and Psychology 6: 547-569.

Lyons, J. (2011). Circularity, Reliability, and the Cognitive Penetrability of Perception. Philosophical Issues 21, The Epistemology of Perception: 289-311.

Macpherson, F. (2012). Cognitive penetration of colour experience: Rethinking the issue in light of an indirect mechanism. Philosophy and Phenomenological Research 84 (1): 24-62.

Macpherson, F. (2017). The relationship between cognitive penetration and predictive coding. Consciousness and Cognition 47: 6-16.

Matthews, W. and W. Meck (2016). Temporal cognition: connecting subjective time to perception, attention, and memory. Psychological Bulletin 142 (8): 865-907.

Meck, W. and C. Malapani (2004). Neuroimaging of interval timing. Cognitive Brain Research 21: 133-137.

Merino-Rajme, C. (2014). A quantum theory of felt duration. Analytic Philosophy55 (3): 239-275. 
Michotte, A. (1946/1963). The Perception of Causality. (T. Miles and E. Miles, trans.) London: Methuen.

Montemayor, C. (2013). Minding Time: A philosophical and theoretical approach to the psychology of time (Leiden and Boston: Brill).

Newen, A. and P. Vetter (2017). Why cognitive penetration of our perceptual experience is still the most plausible account. Consciousness and Cognition 47: 26-37.

Newtson, D., G. Engquist, and J. Bois (1977). The objective basis of behavior units. Journal of Personality and Social Psychology 35 (12): 847-862.

Phillips, Ian (2014). The temporal structure of experience. In Lloyd and Arstila, eds. Subjective time: the philosophy, psychology, and neuroscience of temporality (Cambridge, MA: MIT Press): 139-158.

Poppel, E. (2004). Lost in time: a historical frame, elementary processing units and the 3-second window. Acta Neurobiologiae Experimentalis 64: 295-301.

Pylyshyn, Z. (1999). Is vision continuous with cognition? The case for cognitive impenetrability of visual perception. Behavioral and Brain Sciences 22: 341-423.

Quine, W.V.O. (1985). Events and reification. In E. LePore and B. McLaughlin, eds. Actions and Events: Perspectives in the philosophy of Donald Davidson (Oxford: Blackwell): 162-171.

Radvansky, G. and J. Zacks (2017). Event boundaries in memory and cognition. Current Opinion in Behavioral Sciences 17: 133140. 
Raftopoulos, A. (2001). Neural pathways and the theoryladenness of perception. Philosophy of Science 68 (3) Supplement: Proceedings of the 2000 Biennial meeting of the Philosophy of Science Association: S187-S199.

Reynolds, J., J. Zacks and T. Braver (2007). A computational model of event segmentation from perceptual prediction. Cognitive Science (31): 613-643.

Shea, N. (2015). Distinguishing top-down from bottom-up effects. In D. Stokes, M. Matthen, and S. Biggs, eds., Perception and Its Modalities (Oxford: Oxford University Press): 73-91.

Siegel, S. (2012). Cognitive Penetrability and Perceptual Justification. Nous 46 (2): 201-222.

Siegel, S. and N. Silins (2015). The epistemology of perception. In M. Matthen, ed., The Oxford Handbook of Philosophy of Perception (Oxford: Oxford University Press): 781-811.

Sekuler, R., A. Sekuler, and R. Lau (1997). Sound alters visual motion perception. Nature 385: 508.

Shipley, T. and J. Zacks (2008). Understanding Events: From perception to action. (0xford: Oxford University Press).

Schwan, S. and B. Garsoffsky (2008). The role of segmentation in perception and understanding of events. In T. Shipley and J. Zacks, eds. Understanding Events (Oxford: Oxford University Press).

Stokes, D. (2013). Cognitive penetrability of perception. Philosophy Compass 8 (7): 646-663. 
Teigen, K., G. Bohm, S. Bruckmiller, P. Hegarty, and O. Luminet (2017). Long live the king! Beginnings loom larger than endings of past and recurrent events. Cognition 163: 26-41.

Terhune, D., S. Russo, J. Near, C. Stagg, and R. Kadosh (2014). GABA predicts time perception. The Journal of Neuroscience 34 (12): 4364-4370.

Treisman, M. (1963). Temporal discrimination and the indifference interval: implications for a model of the "internal clock". Psychological Monographs 77 (13): 1-31.

Tversky, B., J. Zacks, and B. Hard (2008). The structure of experience. In T. Shipley and J. Zacks, eds. Understanding Events (Oxford: Oxford University Press): 436-464.

Vance, J. (2014). Emotion and the new challenge from cognitive penetrability. Philosophical Studies 169: 257-283.

Vance, J. (2015). Cognitive Penetration and the Tribunal of Experience. Review of Philosophy and Psychology 6: 641-663.

Watanabe, K. and S. Shimojo (2005). Crossmodal Attention in Event Perception. In L. Itti, G. Rees, J. Tsotsos, eds. Neurobiology of attention (Amsterdam: Elsevier): 538-543.

Wearden, J. (2005). Origines et developpement des theories d'horloge interne du temps psychologique [Origin and development of internal clock theories of time]. Psychologie Francaise 50: 7-25.

Zacks, J. (2001). Scaling up from atomic to complex events. Behavioral and Brain Sciences 24 (5): 9009-910.

Zacks, J. and B. Tversky (2001). Event structure in perception and cognition. Psychological Bulletin 127 (1): 3-21. 
Zacks, J., N. Speer, K. Swallow, T. Braver, and J. Reynolds (2007). Event perception: a mind/brain perspective. Psychological Bulletin 133 (2): 273-293.

Zacks, J., B. Tversky, and G. Iyer (2001). Perceiving, remembering, and communicating structure in events. Journal of Experimental Psychology: General 130 (1): 29-58. 